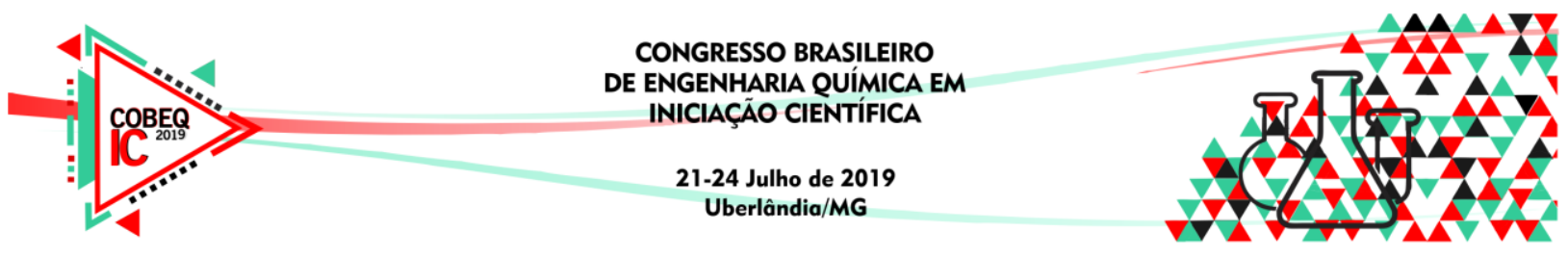

\title{
USO DO MÉTODO ESPECTROFOTOMÉTRICO PARA A DETERMINAÇÃO DE ENXOFRE EM FERTILIZANTES.
}

\author{
Y. L. M. COSTA ${ }^{1}$, L. M. R. SANTOS ${ }^{1}$, D. V. B. $\operatorname{CAMPOS}^{2}$, B. B. $\operatorname{MATTOS}^{2}$ e G. S. \\ MARTINS $^{2}$ \\ ${ }^{1}$ Universidade de Vassouras, graduação em Engenharia Química \\ ${ }^{2}$ Embrapa Solos \\ E-mail para contato: lopess.yo@gmail.com
}

\begin{abstract}
RESUMO - O método mais utilizado para a determinação de enxofre (S) em fertilizantes é o gravimétrico, contudo, o método espectrofotométrico pode ser uma alternativa, sendo o procedimento que utiliza a luz para medir a concentração química de qualquer espécie, de forma mais rápida e simples. O trabalho teve como objetivo determinar os teores de enxofre através do método espectrofotométrico em oito fertilizantes contendo $\mathrm{S}$ em sua composição, comparando com a garantia e os limites aceitáveis. A curva padrão apresentou $\mathrm{R}^{2}=0,9967$. Visto que os teores de $\mathrm{S}$ determinados nos fertilizantes pesquisados estão acima da garantia, todos os fertilizantes pesquisados foram considerados satisfatórios em relação ao teor de enxofre. O método espectrofotométrico apresenta grande potencial para análise de $\mathrm{S}$ em fertilizantes.
\end{abstract}

\section{INTRODUÇÃO}

O enxofre (S) é um elemento essencial à vida na Terra, sendo alguns de seus compostos de grande importância biológica (Martins et al., 2003). Ele possui um papel fundamental na síntese de carboidratos, sendo um componente de diversas enzimas, sendo classificado como um macronutriente secundário. O solo fornece enxofre para as plantas, porém para grandes produtividades, se faz necessário o uso de fertilizantes, tal qual o enxofre apresenta-se na forma de sulfato, elementar ou com a combinação de ambos (Marschner, 1988).

Dentre as fontes tradicionais de enxofre, as mais utilizadas são o sulfato de amônio, superfosfato simples e o gesso agrícola (Câmara, 2014). O método mais utilizado para realizar análise de enxofre em fertilizantes é o gravimétrico, contudo, outros métodos podem ser desenvolvidos. O método espectrofotométrico pode ser uma alternativa, sendo definido como o procedimento que utiliza a luz para medir a concentração química de qualquer espécie. Trata-se de método de análise óptico mais usado nas investigações biológicas e físicoquímicas. Esta técnica tornou-se fundamental para a determinação de diagnósticos laboratoriais (Oliveira, 2012).

O presente estudo teve como objetivo avaliar os teores de enxofre em fertilizantes utilizando o método espectrofotométrico. Os fertilizantes escolhidos apresentam teores de S conhecidos. 


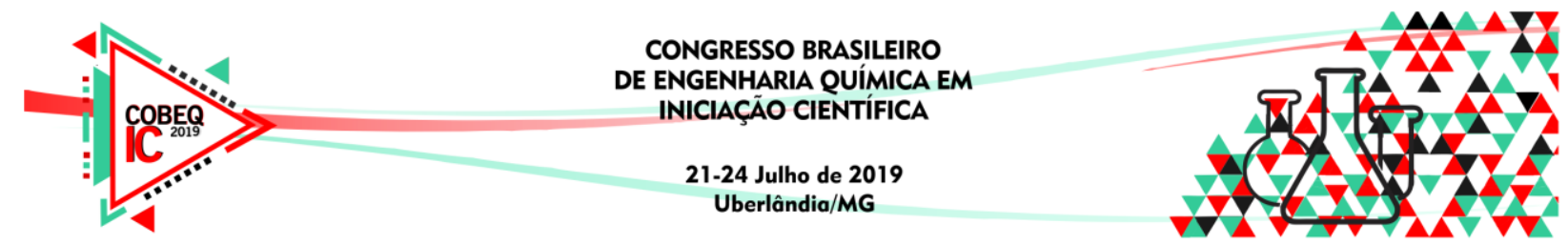

\section{MATERIAIS E MÉTODOS}

As análises de oito amostras foram realizadas em triplicata no laboratório da Embrapa Solos RJ. Onde foram quarteadas e pesadas, de acordo com o manual de métodos para análise de fertilizantes e corretivos do Ministério da Agricultura, Pecuária e Abastecimento (Ministério de Agricultura, Pecuária e Abastecimento, 2017), no Laboratório de Tecnologia em Fertilizantes da Embrapa Solos.

\subsection{Extração com HCl Concentrado}

De acordo com a garantia do fertilizante, as amostras foram pesadas e posteriormente transferidas para um béquer de $250 \mathrm{~mL}$. Foram adicionados $10 \mathrm{~mL}$ de água ultrapura e $10 \mathrm{~mL}$ de $\mathrm{HCl}$ concentrado. Os béqueres foram colocados sobre uma chapa aquecedora a $100{ }^{\circ} \mathrm{C} \mathrm{e}$ aquecidos até próximo o ponto de evaporação, em que as amostras apresentavam uma consistência pastosa. Após o resfriamento, foram adicionados mais $10 \mathrm{~mL}$ de $\mathrm{HCl}$ concentrado e $50 \mathrm{~mL}$ de água ultrapura. Em seguida, as amostras foram aquecidas novamente por 10 minutos, e logo após o aquecimento, foram filtradas através de um funil e papel de filtro com porosidade média para um béquer de $400 \mathrm{~mL}$. Após a filtração, o papel de filtro com o resíduo foi lavado com aproximadamente $200 \mathrm{~mL}$ de água destilada a $85-90^{\circ} \mathrm{C}$, em pequenas porções. O líquido filtrado foi então transferido para um balão volumétrico de 250 $\mathrm{mL}$ e posteriormente avolumado (Ministério da Agricultura, Pecuária e Abastecimento, 2014). A seguir na Tabela 1 , as amostras utilizadas de fertilizantes e suas respectivas garantias.

Tabela 1 - Identificação das amostras contendo S e suas garantias

\begin{tabular}{|c|c|}
\hline Amostras & $\begin{array}{c}\text { Garantia } \\
(\mathbf{\%})\end{array}$ \\
\hline Gesso & 18,5 \\
\hline Superfosfato Simples & 12,5 \\
\hline Sulfato de Amônio & 24,1 \\
\hline Sulfato de Potássio (Agricultura Orgânica) & 18,5 \\
\hline Gesso + MgO & 11,1 \\
\hline Superfosfato Triplo & 1,4 \\
\hline Du Solo 10\% & 2,8 \\
\hline Du Solo 50\% & 10,6 \\
\hline
\end{tabular}

\subsection{Determinação pelo Método Espectrofotométrico}

A determinação do $\mathrm{S}$ foi feita por método espectrofotométrico, adaptado da leitura de amostra feita para extrato de solo na metodologia usada por Xanchão (Dominguez, 2014; Cantarella e Prochnow, 2001)(Sulfato \& Solos, 1973). A análise consistiu no seguinte procedimento. Para cada amostra, foi pipetado $1,00 \mathrm{~mL}$ de cada balão volumétrico e adicionado mais $9 \mathrm{~mL}$ de água ultrapura, em seguida avolumada em um balão volumétrico de $100 \mathrm{~mL}$. Posteriormente, foram pipetados $10 \mathrm{~mL}$ da solução diluída em um béquer de $50 \mathrm{~mL}$, 


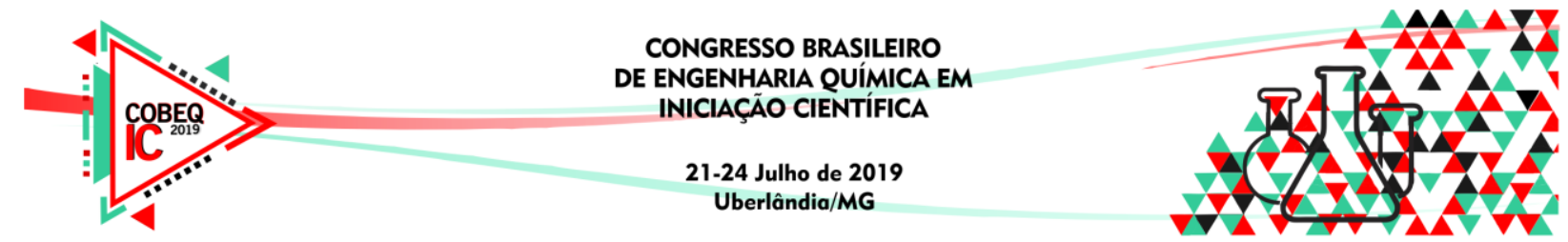

onde foram adicionados mais $1 \mathrm{~mL}$ de solução semente-ácida e $0,5 \mathrm{~g}$ de $\mathrm{BaCl}_{2} .2 \mathrm{H}_{2} \mathrm{O}$ macerado e seco. Esperou-se 1 minuto, logo após as amostras foram agitadas manualmente até a dissolução dos cristais. As leituras das amostras foram feitas após 4 minutos da dissolução dos cristais em espectrofotômetro (NOVA 1600 UV) a $420 \mathrm{~nm}$. O aparelho foi zerado com a solução em branco (ponto $0 \mathrm{mg} \mathrm{L}^{-1}$ de S-SO${ }_{4}^{-2}$ da curva-padrão).

Para a determinação do teor (\%) de enxofre presente nas amostras de fertilizantes, utilizou a Equação 1.

$$
S(\%)=\frac{\left(\left(\frac{L-b}{a}\right) \times d\right)}{m} \times 100
$$

Onde:

L - Leitura da amostra, em absorvância.

a - Coeficiente angular da reta dos padrões.

$\mathrm{b}$ - Coeficiente linear da reta dos padrões.

d - Fator de diluição do extrato (quando não foi necessária a diluição, considerou-se $d=1$ ).

m - Massa pesada do fertilizante em mg.

Construção da curva-padrão: A partir de uma solução padrão de $100 \mathrm{mg} \mathrm{L}^{-1}$ de $\mathrm{S}$. Foram pipetados $0,5,10,15,20,25,50 \mathrm{~mL}$ dessa solução em balões volumétricos de 100 $\mathrm{mL}$, onde acrescentou-se mais $4 \mathrm{~mL}$ de $\mathrm{HCl}$ concentrado e completou-se o volume de cada balão com água ultrapura. Para a construção da curva-padrão, foram pipetadas $0,5,10,15$, 20, 25, $50 \mathrm{~mL}$ das soluções-padrão e adicionado novamente mais $4 \mathrm{~mL}$ de $\mathrm{HCl}$ concentrado e avolumado com água ultrapura em um balão volumétrico de $100 \mathrm{~mL}$, , obtendo soluções com concentrações de sulfato equivalentes a 5, 10, 15, 20, 25 e $50 \mathrm{mg} \mathrm{L}^{-1}$, conforme a Figura 1.

Figura 1 - Curva-padrão para a determinação de S em fertilizantes.

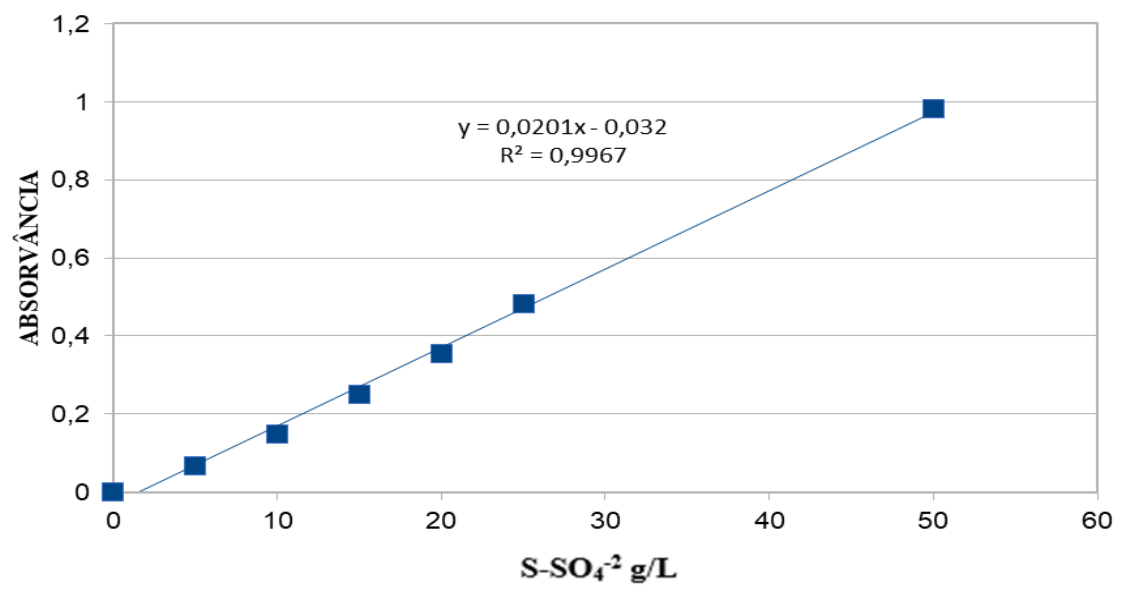




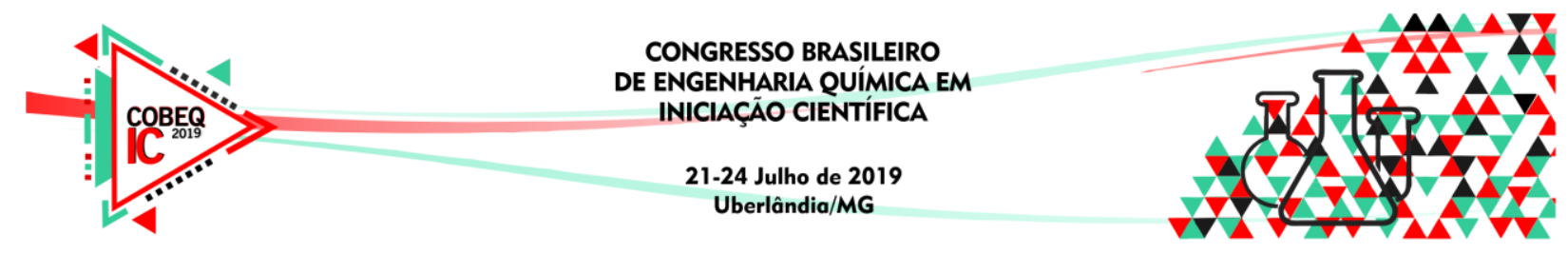

\subsection{Cálculo dos Limites Toleráveis}

Com base na garantia de cada fertilizante, foi calculada individualmente a tolerância e os limites aceitáveis dos teores de enxofre, com base na Instrução Normativa $\mathrm{n}^{\mathrm{o}}: 46$ (Ministério da Agricultura, Pecuária e Abastecimento, 2016). Os fertilizantes foram classificados como satisfatórios, quando o teor determinado na análise de $\mathrm{S}$ estava dentro dos limites aceitáveis, e insatisfatório, caso o teor encontrado fosse inferior aos limites aceitáveis.

\section{RESULTADOS E DISCUSSÃO}

Com base nas análises de nutrientes em fertilizantes, é comum ocorrer uma variabilidade em seus teores. Para sua comercialização pela legislação, são admitidas tolerâncias para cada fertilizante. A tabela a seguir, mostra o teor de enxofre encontrado em cada amostra de fertilizante através do método espectrofotométrico, suas respectivas garantias, tolerância e o limite aceitável.

Tabela 2 - Teor de S, garantia, tolerância e limites aceitáveis de fertilizante contendo enxofre.

\begin{tabular}{|c|c|c|c|c|}
\hline Fertilizantes & $\begin{array}{c}\text { Garantia } \\
(\boldsymbol{\%})\end{array}$ & $\begin{array}{c}\text { Tolerância } \\
(\boldsymbol{\%})\end{array}$ & $\begin{array}{c}\text { Limite Aceitável } \\
(\mathbf{\%})\end{array}$ & $\begin{array}{c}\text { Teor S } \\
(\boldsymbol{\%})\end{array}$ \\
\hline Gesso & 18,5 & 1,3 & 17,2 & 20,2 \\
\hline Superfosfato Simples & 12,5 & 1,1 & 11,4 & 11,6 \\
\hline Superfosfato Triplo & 1,4 & 0,255 & 1,145 & 1,5 \\
\hline Sulfato de Amônio & 24,1 & 1,5 & 22,6 & 25 \\
\hline $\begin{array}{c}\text { Sulfato de Potássio (Agricultura } \\
\text { Orgânica) }\end{array}$ & 18,5 & 1,3 & 17,2 & 20,9 \\
\hline Gesso + MgO & 11,1 & 1,04 & 10,06 & 12,2 \\
\hline Du Solo 10\% & 2,8 & 0,45 & 2,35 & 3,6 \\
\hline Du Solo 50\% & 10,6 & 1,52 & 9,08 & 12,8 \\
\hline
\end{tabular}

Dentre os fertilizantes com teores mais elevados de enxofre, os produtos Gesso, Sulfato de Amônio, Sulfato de Potássio, Gesso+MgO e Du Solo 50\% apresentaram valores acima da garantia para comercialização, com 20,2 \%, 25,0 \%, 20,9 \%, 12,2 \% e 12,8 \%, respectivamente. O produto Superfosfato Simples, com teor de $S$ igual a 11,6\%, foi abaixo da garantia, mas acima do limite aceitável de 11,4\%, de acordo com a Tabela 2.

Para os fertilizantes com baixo teor de S, os produtos Superfosfato Triplo e Du Solo10\% apresentaram teores próximos à garantia, sendo considerados satisfatórios, como mostra a Tabela 2.

Através dos resultados obtidos, os fertilizantes avaliados, apresentaram teores de $\mathrm{S}$ considerados satisfatórios, demonstrando que o método espectrofotométrico pode ser utilizado em fertilizantes que contenham $\mathrm{S}$ na forma de sulfato.

Com intuito de validar o método, os resultados apresentados através do mesmo, devem ser comparados com os do método gravimétrico, que é o método padrão recomentado pelo Ministério da Agricultura, Pecuária e Abastecimento. 


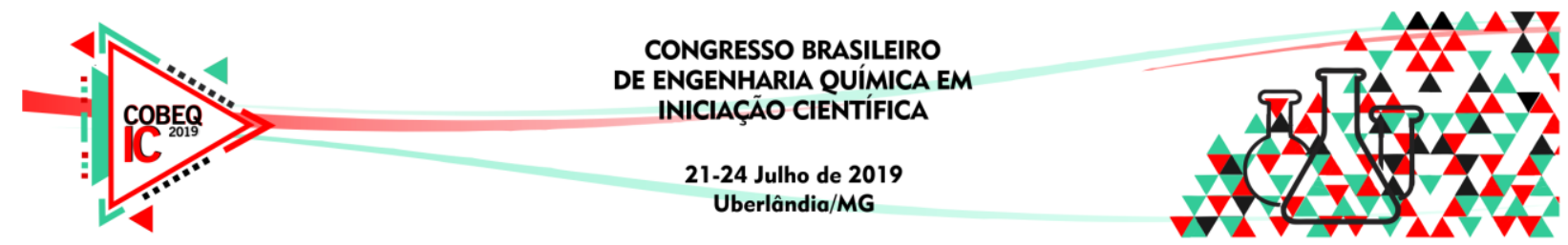

\section{CONCLUSÃO}

Visto que os teores de $\mathrm{S}$ determinados nos fertilizantes pesquisados estão acima da garantia, todos os fertilizantes pesquisados foram considerados satisfatórios em relação ao teor de enxofre. $\mathrm{O}$ método espectrofotométrico apresenta grande potencial para análise de $\mathrm{S}$ em fertilizantes.

\section{REFERÊNCIAS}

Câmara, G. M. de S. (2014). Informações Agronômicas. International Plant Nutrition Institute, (147), 1-9. https://doi.org/10.1186/1749-8090-6-114

Martins, C. R., Pereira, P. A. de P., Lopes, W. A., \& de Andrade, J. B. (2003). Ciclos Globais de Carbono, Nitrogênio e Enxofre: a Importância da Química da Atmosfera. Comunicata Scientiae, 1(2), 28-41.

Ministério da Agricultura, Pecuária e Abastecimento, M. da A. P. e A. (2014). Manual de métodos analíticos oficiais para fertilizante e corretivos, 220. https://doi.org/10.1093/trstmh/trw058

Ministério da Agricultura, Pecuária e Abastecimento, M. da A. P. e A. (2016). Instrução Normativa $\mathrm{N}^{\circ} 46$, De 22 De Novembro De 2016., 1-44. Retrieved from http://www.agricultura.gov.br/assuntos/insumos-agropecuarios/insumosagricolas/fertilizantes/legislacao/in-46-de-22-11-2016-fert-minerais-dou-7-1216.pdf

Ministério de Agricultura, Pecuária e Abastecimento, M. da A. P. e A. (2017). Manual de procedimentos para laboratório.

Oliveira, M. V. P. de. (2012). Aplicações De Estudos Bioquímicos Quantitativos Em Ciências Biológicas E Da Saúde. Estudos Bioquímicos Quantitativos, 2, 99-127.

Sulfato, D. D. E., \& Solos, E. M. (1973). Capítulo 14, 0-5.

MARSCHNER, H. Mineral nutrition of higher plants, 2. Ed., Academic Press, 1988. 